\title{
A SPEED-UP GEOMETRIC CHANGE DETECTION ALGORITHM FOR VECTOR SURFACE FEATURE SET
}

\author{
Lining. Zhu ${ }^{1 *}$, Chengming. Li ${ }^{1}$, Li. Liu ${ }^{1}$, Jianming. Shen ${ }^{1}$, Lina. Yang ${ }^{2}$, Zhendong. Liu ${ }^{1}$ \\ ${ }^{1}$ Institute of Cartography and Geographic Information System, Chinese Academy of Surveying and Mapping, Beijing, China - \\ zhu_li_ning@163.com, cmli@casm.ac.cn,372319261@qq.com, jianmingsh@126.com, lzdgis08@126.com \\ ${ }^{2}$ College of Surveying and Geography, LiaoNing Technical University, Liaoning, China - 2533637007@qq.com
}

\section{Commission IV, WG IV/6}

KEY WORDS: Vector Surface Feature Set, Candidate Set, Geometric Change Detection, Geometric Shrinkage, Precise Query, NewMap

\begin{abstract}
:
At present, most of the researches on geometric change detection of vector data, they store the change detection results in the database, so they pay more attention to the accuracy of results, but not to the speed of processing. Nowadays, many applications require real-time change detection on vector data and rapid presentation of the result. Although the existing algorithms use spatial index technology to improve the processing speed, the processing time is still beyond the range that people can bear. In order to reduce processing time, this paper takes the vector surface feature set as the research object, trying to reduce the redundancy of the candidate set that seriously affects the efficiency of change detection. Based on the regular use of spatial index created with geometric Minimum Bounding Rectangle, this paper uses geometric shrinkage technique and precise query technique to reduce the size of the candidate set for detection, so as to achieve the goal of speeding up. Finally, using five years of farmland data and resident data from Ezhou City, Hubei Province, China, a change detection experiment was conducted. The experiment proved that the geometric shrinkage and precise query techniques can effectively improve the processing speed.
\end{abstract}

\section{INTRODUCTION}

Change detection technology is based on computer image processing system, to identify changes in different periods of the target or phenomenon state. The change detection of geographic information data mainly includes remote sensing image change detection and vector data change detection. In recent years, the change detection technology based on remote sensing image has made considerable achievements, and the change detection based on vector data has gradually become a hot topic at home and abroad. For example, Zhao Yong (Zhao, 2006) studied the statistical method based on area change, used the polygon intersection of vector data and the change pixels of grid data to calculate the change area respectively; Guo Li (Guo, 2009) used area similarity to study the real body matching based on the area similarity; Deng Xiaolian (Deng, 2006), Zeng Zifang (Zeng, 2013), Wang Liyun (Wang, 2014) studied the method of land use change detection based on variable vector analysis; Zhang Wei (Zhang, 2012) used Canadian FME software to obtain the statistical information of change information and changes directly; Sha Yukun (Sha, 2012) used the intersection and difference of the set to establish a change detection model of the vector data group of three elements; Qiu Bo (Qiu, 2013) made a very careful division of the basic change types of the facial elements in the change detection of the vector surface elements, and the focus of the study was on the accuracy of the change detection. Wang Wenjie (Wang, 2013) put forward the 1:1 geometric change detection method of multi feature similarity synthesis for space-time line and surface element, and a complex change detection method for multiple entities was proposed on this basis; Wang Huai (Wang, 2014) proposed a geometric matching similarity change detection method for geographical conditions, and Chen Zhanlong (Chen, 2016) gave a method of geometric similarity measurement; Liu Jie (Liu, 2017) designed a geographical factor change detection method based on different temporal homologous reference data.

At present, more and more applications need vector change detection technology, and the processing speed of vector change detection is required in some applications. Like the big data center in the construction of smart city, it needs to provide a tool to quickly detect the changes of the two phases of data. In the national census geography, it is also necessary to find out the change of land class as soon as possible. However, existing research is difficult to meet their speed requirements, as these studies pay more attention to the accuracy of the results, rather than the processing speed.

In the vector data change detection, the determination of the detection candidate set has a great influence on the efficiency of the change detection. Most of the above studies speed up the detection of the candidate set by setting up the spatial index. Like Wang Wenjie (Wang, 2013) and Qiu Bo (Qiu, 2013), they use the MBR (Minimum Outsourced Rectangle) to select the detection candidate set, but the quality of the generated detection candidate set is not much concerned. This leads to two problems. Firstly, using the MBR to determine the detection candidate set will, to some extent, cause redundancy in the detection candidate set, because the intersection of the two geometric MBR does not mean that the geometry itself will intersect; Secondly, in actual production, due to the influence of various errors originating from the machine, people and so on, the two actual adjacent geometries will show a phenomenon of boundary crossing on the data, which will also cause

\footnotetext{
* Corresponding author
} 
redundancy of detection candidate sets to some extent. Both of these conditions reduce the quality of the selected detection candidate set and the speed of change detection.

Aiming at the above two cases, this paper uses the geometric shrinkage technique and the precise query after MBR fuzzy query to reduce the redundancy of the detection candidate set, thus improving the efficiency of change detection. In addition, the vector data includes point, lines, and surface. In this paper, only the surface data is studied, point and line data are not considered temporarily.

\section{GEOMETRIC CHANGE DETECTION}

In this paper, the geometric state is divided into three types: new, disappeared, and unchanged. The algorithm this paper designed is mainly to find out the new added or disappeared geometries of two tenses. The following will introduce two key techniques this paper introduced to improve the processing speed: the geometric shrinkage technique and precise query technique, and the flow of the improved change detection algorithm in this paper.

\subsection{Geometric Shrinkage Technique}

Geometric shrinkage, as shown in Figure 1, is a kind of geometric transformation. In this paper, this technique is used to eliminate the actual disjoint geometries but the data crossed caused by artificial and machine errors. As shown in Figure 2, geometry 'a' and geometry 'b' are actually a kind of adjacency relationship, but because of the influence of error, there is a boundary crossing between them at the data level. Assume that the intersection distance is ' $\mathrm{d}$ ', if we set an error range ' $\beta$ ' (' $\beta$ ' $>=$ ' $d$ '), then shrink the geometry ' $a$ ' to obtain the geometry ' $a_{\mathrm{s}}$ ' (shrinking distance is $\beta$ ). Now geometry ' $a_{\mathrm{s}}$ ' and geometry ' $b$ ' have no boundary crossing phenomenon, the effect of the error is eliminated.

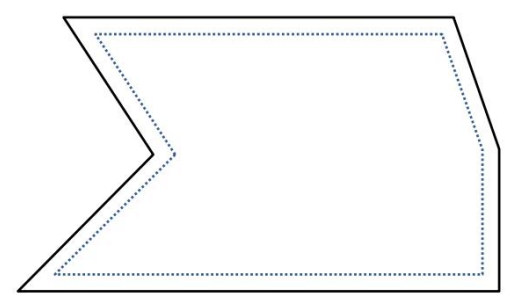

Figure 1. Geometric shrinkage

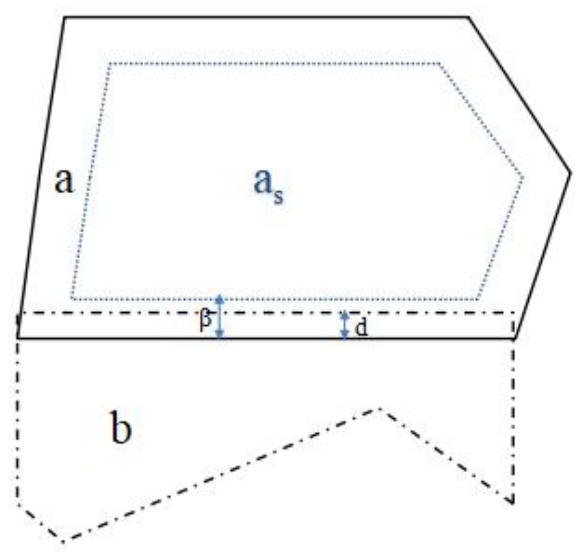

Figure 2. Boundary crossing caused by error

\subsection{Precise Query Technique}

When searching for a candidate set, most existing studies use fuzzy query, that is, using the detected geometric Minimum Bounding Rectangle to filter the corresponding detection candidate set from the reference data set. After all, it is more convenient to create a spatial index using the geometric Minimum Bounding Rectangle. However, the fuzzy query does not ensure that each geometry in the detection candidate set intersects with the geometry to be detected, as the intersection of the two geometric Minimum Bounding Rectangle does not mean that the two geometries are intersected. In order to ensure that each geometry in the candidate set is intersected with the geometry to be detected, this paper first finds out a rough detection candidate set based on the regular use of spatial index created with geometric Minimum Bounding Rectangle, then introduces the precise query technique, which is to further filter the rough detection candidate set and remove the items that are not intersected with the geometry to be detected. The candidate set after cleaning is the candidate set that will eventually participate in the computation.

\subsection{Improved Geometric Change Detection Algorithm}

After introducing the geometric shrinkage technique and precise query technique, the geometric change detection algorithm flow is as follows. The third step in the algorithm uses the geometric shrinkage technique, and the fifth step uses the precise query technique.

Step 1, prepare the data and parameters required by the algorithm, including the surface feature set ' $A$ ', the surface feature set ' $\mathrm{B}$ ', and the coordinate tolerance ' $\beta$ '.

Step 2, build the R-tree spatial index for the set ' $A$ ' and 'B', now we begin to detect changes in feature set ' $\mathrm{A}$ ' relative to feature set ' $\mathrm{B}$ '.

Step 3, traverse the surface feature set ' $A$ ', take out a geometry ' $a$ ' in set ' $A$ '. If there is no geometry desirable, skip to step 12 .

Step 4 , the geometry ' $a$ ' is shrunk to obtain the geometry ' $a_{s}$ ' and the shrinkage distance is ' $\beta$ '.

Step 5, use fuzzy query to get the rough detection candidate set 'Br', ensure that each geometry in 'Br', its Minimum Bounding Rectangle is intersected with the Minimum Bounding Rectangle of geometry ' $a_{\mathrm{s}}$ '.

Step 6, traverse the set ' $\mathrm{Br}$ ', remove the geometry that does not intersect with the geometry ' $\mathrm{a}_{\mathrm{s}}$ ', and the remaining geometries in the set ' $\mathrm{Br}$ ' constitute the detection candidate set ' $\mathrm{Ba}$ ' for the geometry ' $a$ '.

Step 7, if the set ' $\mathrm{Ba}$ ' is empty, it indicates that the geometry ' $\mathrm{a}$ ' is completely changed, store geometry ' $a$ ' in the result set ' $R$ ', skip to the step 3, continue to process the next geometry in the surface feature set 'A'; otherwise, continue to execute downward.

Step 8, traverse the set ' $\mathrm{Ba}$ ', to see if there is a certain geometry equals to the geometry ' $a$ ' within the coordinate tolerance ' $\beta$ '. If it exists, then the geometry ' $a$ ' does not change, skip to the step 3 , and continue to process the next geometry in the surface feature set ' $A$ '; if it does not exist, continue to execute downward. 
Step 9, each geometry in set ' $\mathrm{Ba}$ ' intersects with geometry ' $\mathrm{a}$ ', and all intersection results are merged into a geometry ' $u$ '.

Step 10, the geometry ' $u$ ' and geometry ' $a$ ' perform the different operation to obtain the geometry ' $r$ '. The geometry ' $r$ ' is the change part of the geometry ' $a$ ', and store it in the result set ' $R$ '.

Step 11, whether the surface feature set ' $A$ ' is traversed or not, if not, execute the step 3 .

Step 12, exchange the roles of surface feature set ' $A$ ' and surface feature set 'B', repeat step 3 to step 11 .

Step 13 , within the range of the coordinate tolerance ' $\beta$ ', clear the self-intersecting part of the geometry, and remove the narrow line surface in the result set ' $R$ '. The result set ' $R$ ' after cleaning is the change detection result.

\section{EXPERIMENT}

\subsection{Experimental Conditions}

This paper uses c++ language to realize the above improved algorithm. In the algorithm implementation, the geometric operator provided by the NewMap software of Chinese Academy of Surveying and Mapping is called, including geometric intersection, difference, union, comparison and shrinkage. In addition, to verify the effectiveness of the improvements proposed in this paper, an algorithm that only uses precise query technique and an algorithm that uses neither geometric shrinkage technique nor precise query technique are also realized as experimental references.

The experimental data uses farmland data and resident data from 2011 to 2015 in Ezhou City, Hubei Province, China. The annual farmland factor number is between 9500 and 11000 , and the annual resident land factor number is between 5800 and 7000. The experimental machine is the HP ZBook 15 G3 mobile workstation. There are 18 experimental values for the tolerance $\beta$, including $1 \mathrm{~m}, 0.7 \mathrm{~m}, 0.5 \mathrm{~m}, 0.3 \mathrm{~m}, 0.2 \mathrm{~m}, 0.1 \mathrm{~m}$, $0.07 \mathrm{~m}, 0.05 \mathrm{~m}, 0.03 \mathrm{~m}, 0.01 \mathrm{~m}$, and $0.007 \mathrm{~m} .0 .005 \mathrm{~m}, 0.003 \mathrm{~m}$, $0.001 \mathrm{~m}, 0.0007 \mathrm{~m}, 0.0005 \mathrm{~m}, 0.0003 \mathrm{~m}$, and $0.0001 \mathrm{~m}$. The three experimental algorithms are respectively an algorithm that uses two kinds of improvement measures, an algorithm that uses only the precise query improvement measures, and an algorithm that dose not use both of the two improvement measures. In the five-years of farmland data, change is detected in any two years, and so is the resident data. Each algorithm collects 180 sample data for each type of land, totaling 1080 samples. The sample collection information includes the processing time, the number of change detection results and the change detection results.

\subsection{Analysis of Results}

Processing the sample data to obtain the average processing time of each algorithm for different types of land under different tolerances, and then make a comparison. The comparison results of the farmland data are shown in Figure 3, the comparison results of the resident data are shown in Figure 4, the abscissa stands for the tolerance $\beta$ (in meters), the ordinate represents the average time (in seconds). As can be seen from Figure 3 and Figure 4, the algorithm that uses the geometric shrinkage and precises query improvement measures takes the least time, the algorithm that does not use any improvement measures takes the most time.

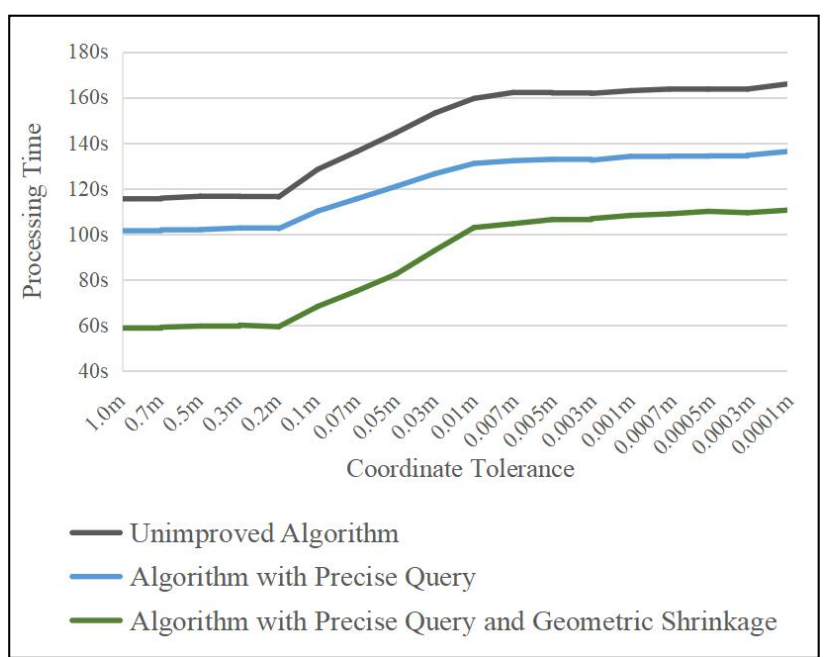

Figure 3. Average processing time comparison of farmland data

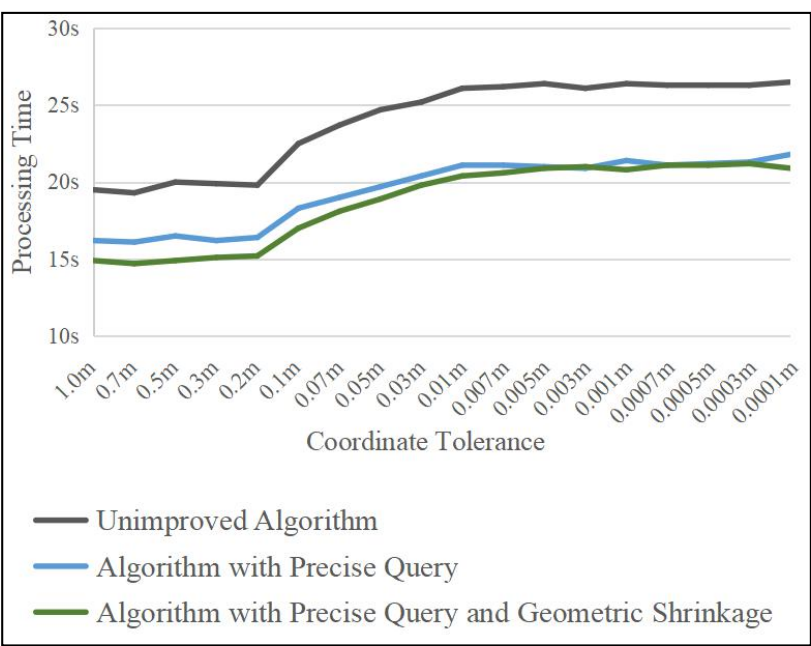

Figure 4. Average processing time comparison of resident data

From Figure 3 and Figure 4, we also can see that as the setting value of the tolerance $\beta$ becomes smaller and smaller, the geometric shrinkage technique plays a smaller role. In fact, when the tolerance setting is relatively small, the geometry shrinkage technique has even played a counter-productive role. Since the average value reflects an overall trend, Figure 3 and Figure 4 do not reflect this situation well. In order to display this situation more intuitively, the comparison of the processing time of all samples of the farmland data is given.

In Figure 5, the abscissa still represents the coordinate tolerance, and the ordinate stands for the difference in processing time that the algorithm with improved measure subtracts the algorithm without any improvement. The difference in processing time of the algorithms for any improvement measures can be seen from Figure 5. It can be seen from Figure 5 that the processing time of algorithm using improvement measures is lower than the algorithm without any improvement measure. However, when the coordinate tolerance is small, the algorithm that introduces both geometric shrinkage and precise query techniques takes more time than the algorithm that only introduces the precise query technique. This paper collects the farmland data samples that geometric shrinkage technique plays a counter-productive role, and shows the result in Figure 6. The abscissa represents the coordinate tolerance, and the ordinate represents the number of samples. It can be seen from Figure 6 that the samples with geometric shrinkage reaction mainly fall in the area where the 
coordinate tolerance is small. This is mainly because the data collection accuracy of the farmland data is not high. If we set the coordinate tolerance too small during calculation, the influence of the error cannot be eliminated, and the significance of introducing the geometric shrinkage technology is also lost.

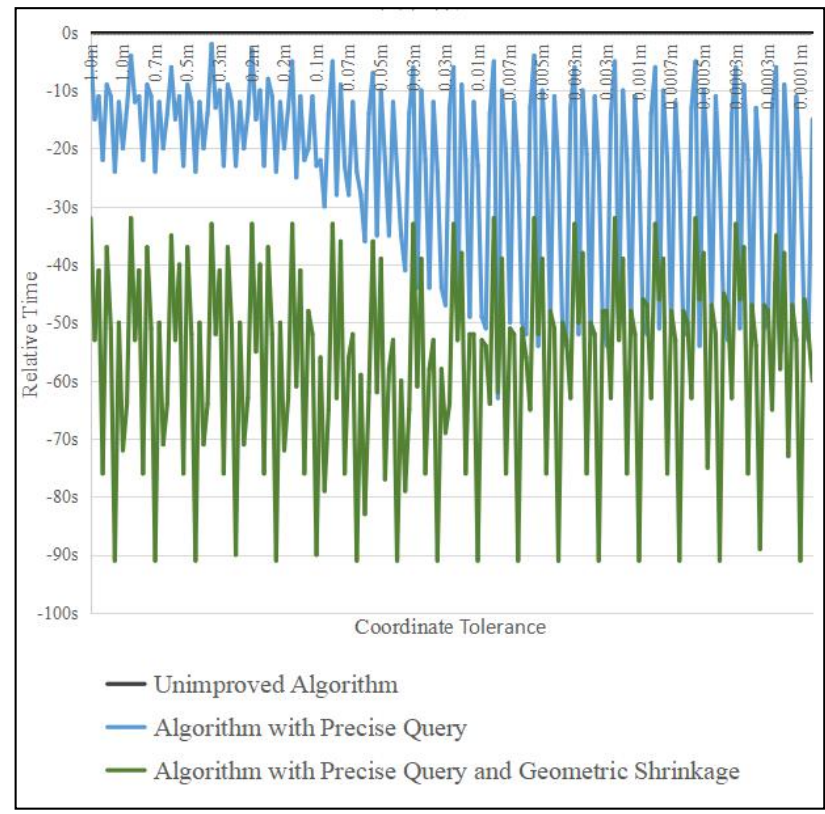

Figure 5. Processing time of all samples of farmland data

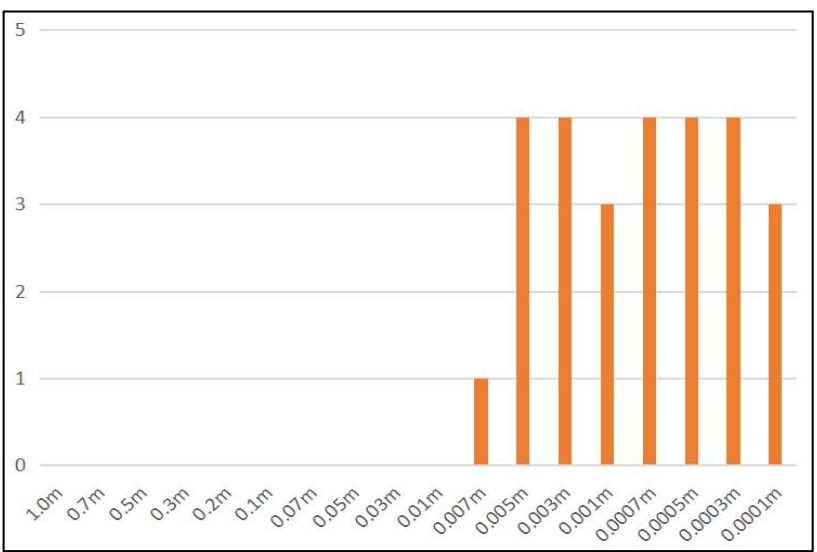

Figure 6. Geometric shrinkage technique failure distribution

\section{CONCLUSION}

In order to improve the efficiency of the geometric change detection of the vector surface feature set, two improvement measures are proposed in this paper, including geometric shrinkage technique and precise query technique, without affecting the accuracy of the result. Both of improvement measures are aimed at speeding up by reducing the redundancy of the candidate set for detection. The experiment also verified the effectiveness of the improvement measures.

However, the geometric shrinkage technique is affected by the coordinate tolerance. If the value of the coordinate tolerance is not appropriate, it will affect the processing speed. In practice, the tolerance should be set according to the data accuracy requirement of the application, and the greater the better, without exceeding the accuracy requirement.
Although the measures proposed in this paper can effectively improve the speed of geometric change detection, there is still room for improvement. The future work of this paper is to continue to improve the speed of geometric change detection.

\section{ACKNOWLEDGEMENTS}

The research was funded by the project supported by the National Key Research and Development Program of China (Grant No. 2016YFF0201305)

\section{REFERENCES}

Chen, Z., Xu, Y., \& Xie, Z.2016. Discussion on geometrical similarity measurement of vector planar elements

. Science of Surveying and Mapping, 41(5), pp. 105-110.

Deng, X.L.2006. Researches on land use change detection based on change vector analysis method. Doctoral dissertation, institute of remote sensing and digital earth chinese academy of sciences.

Guo, L., Zheng, H., \& Wang, H.2009. Research on surface vector space data matching technology. Hydrographic Surveying and Charting, 29(3), pp. 12-15.

Liu, J. 2017. Geographical Element Change Detection Method Oriented to Geographical Conditions Monitoring. Geospatial Information, 15(5), pp. 32-34

Qiu, B.2013. The research of change detection methods for vector surface elements. Doctoral dissertation, shandong university of science and technology.

Ren, J., Ma, W., \& Mu, L.2015. Extraction method of vector data change quantity in the geographical conditions census. Geospatial Information, 13(5), pp. 6-9+183.

Sha, Y.K.2012. The research of vector data change detection method based on triples. Doctoral dissertation, shandong agricultural university.

Wang, H., Gao, X., Fan, W., \& He, Z.2014. Study on the geographical situation elements spatio-temporal change. Bulletin of Surveying and Mapping, 11, pp. 84-97+119.

Wang, L., Li, Y., \& Wang, Y.2014. Research on Land Use Change Detection Based on an Object-oriented Change Vector Analysis Method. Journal of Geo-Information Science, 16(2), pp. 307-313.

Wang, W.J.2013. The research of geometric change detection methods for spatio-temporal vector data. Doctoral dissertation, lanzhou jiaotong university.

Zeng, Z.F.2013. Change detection research for remote sensing image land use based on the change vector analysis method. Doctoral dissertation, chongqing jiaotong university.

Zhang, W.2012. A study of the method of extraction and statistics of changed information in vector update. Geomatics \& Spatial Information Technology, 35(11), pp. 91-93.

Zhao, Y.2006. A study of the technology of areal ground features change detection based on vector map data. Doctoral dissertation, information engineering university. 